\title{
Antrochoanal polyp
}

\begin{abstract}
Nasal polyps are usually classified into antrochoanal polyps and ethmoidal polyps. Antrochoanal polyps arise from the maxillary sinuses and are the much less common; ethmoidal polyps arise from the ethmoidal sinuses.

The antrochoanal polyp, a benign solitary polypoid lesion, usually originates in a maxillary sinus, filling and enlarging the sinus cavity with no bone destruction. It passes through the ostium of the sinus into the choana, and from there into the posterior nasopharynx. The soft tissue mass usually does not erode or destroy soft tissue or bony structures. In this process, its radiologic findings are usually characteristic (5). They occur more commonly in children and young adults, and they are mostly unilateral. The etiopathogenesis of ACPs is unclear. Nasal obstruction and nasal drainage are the commonest presenting symptoms. The differential diagnosis should include the causes of unilateral nasal obstruction.
\end{abstract}

Keywords: antrochoanal polyp, choana, CT scan, nasal obstruction, maxillary sinus, treatment
Volume 9 Issue 2 - 2018

\section{Sushila B Ladumor,' Hiba Esmayil \\ 'Consultant Radiologist, Hamad Medical Corporation, Assistant Professor, Clinical Radiology, Weil Cornel Medical College, Qatar \\ ${ }^{2}$ II Radiology Resident, Hamad Medical Corporation, Clinical Imaging, Qatar}

Correspondence: Sushila B Ladumor, Consultant Radiologist, Clinical Imaging Department, Hamad Medical Corporation, Assistant Professor in Clinical Radiology, Weil Cornel Medical College, Doha, Qatar (WCMC-Q) Email drsbladumor@yahoo.com

Received: August 26, 2017 | Published: March 22, 2018
Abbreviations: ACP, Antrochoanal polyps; CT, Computerized Tomography; PNS, para nasal sinus

\section{Introduction with background}

The antrochoanal polyp, usually a solitary benign growth, arises from the maxillary antrum of a non-allergic patient. It grows by extension from the antrum through its ostium into the middle meatus, then goes into the posterior choana and may extend into the nasopharynx. Antrochoanal polyps are known to otolaryngologists and have been frequently reported in the otolaryngologic literature after Killian's excellent description in $1906 .{ }^{1}$ In the radiologic literature, the antrochoanal polyp has been largely ignored. Since it has unique features which help radiologist to reach diagnosis.

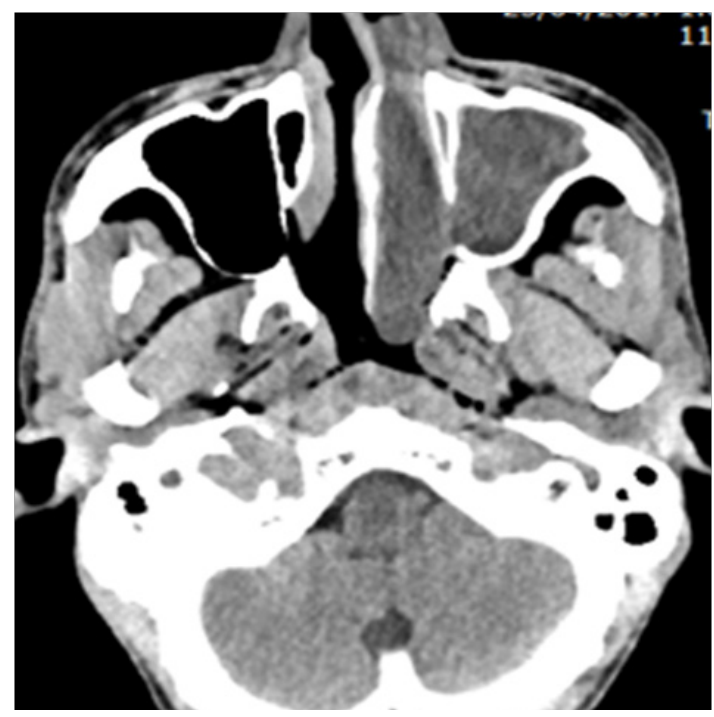

Figure I Non-contrast CT axial image of PNS demonstrates relatively homogenous low density seen filling the left maxillary sinus, protruding into the left nasal cavity through the maxillary ostia. There is total opacification of the left nasal cavity. No associated bony destruction seen. No calcification
Similar, less common, polyps can arise in the sphenoid sinus extending into the nasopharynx: these are termed sphenochoanal polyps. Likewise, there are nasochoanal, frontochoanal, and ethmochoanal polyps.

\section{Case history}

A 46 year old gentleman who presented with left nasal mass with bleeding and ulceration.

\section{Investigation}

CT scan of PNS done with and without intravenous contrast (Figure 1-5).

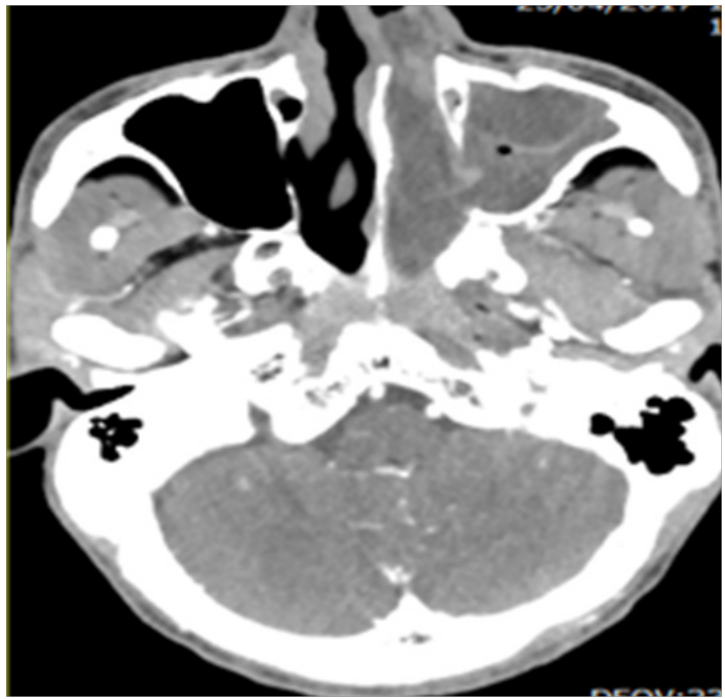

Figure 2 Contrast enhanced CT axial image of PNS demonstrates minimal septal like enhancement of mass with extension of the lesion posteriorly towards the nasopharyngeal airway is seen. 


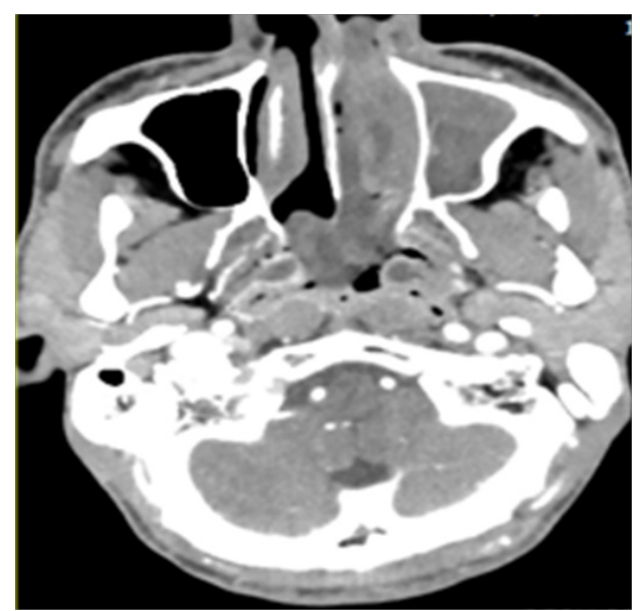

Figure 3 Contrast enhanced CT axial image of PNS demonstrates minimal enhancement of mass with extension of the lesion posteriorly and downwards obliterating the nasopharyngeal airway

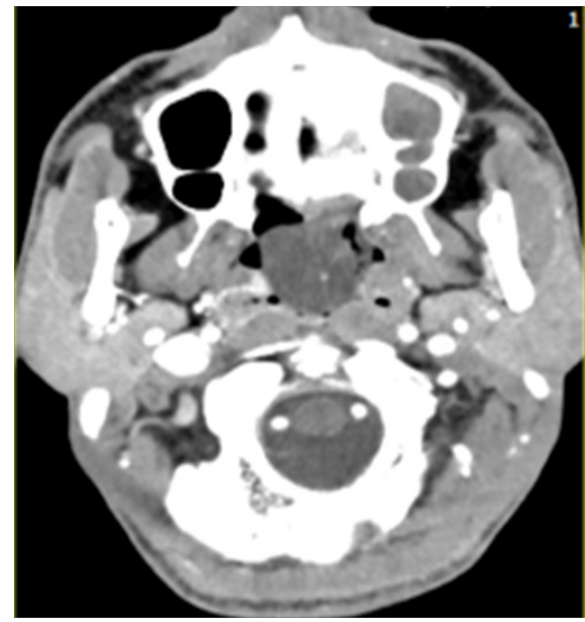

Figure 4 Contrast enhanced CT axial image of PNS demonstrates minimal enhancement of mass with extension of the lesion posteriorly and downwards obliterating the nasopharyngeal airway.

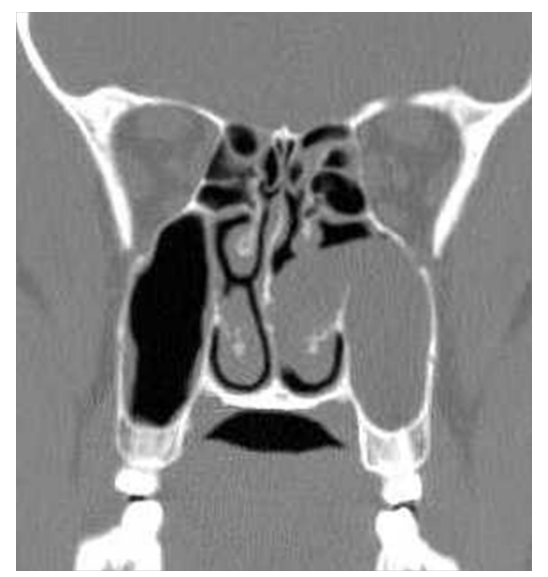

Figure 5 Non-contrast CT, Bone window Coronal reconstruction of PNS demonstrates relatively homogenous mass of soft tissue density is seen in the left maxillary sinus, protruding into the left nasal cavity through the maxillary ostia. Remodeling of bone noted without associated appreciable bony destruction seen.

\section{Findings}

A. Polypoidal lesion expanding within the left maxillary sinus with almost near total opacification into left nasal cavity, extending posteriorly and obliterating the nasopharyngeal airway.

B. No associated bony destruction but rather smooth enlargement of the sinus

C. The lesion displayed soft tissue density mostly non-enhancing lesion, with minimal internal septal like enhancement effacing and inseparable from middle and inferior turbinate

Impression: Above described imaging findings are keeping with antrochoanal polyp.

\section{Discussion}

Antrochoanal polyp, which is also known as "Killian's polyp" is a benign solitary lesion with a mucin density. It arises from the antrum of the maxillary sinus and passes through the sinus ostium into the nasal cavity, choana and goes downwards into the nasopharynx. They are pedunculated and prolapsed pearly white edematous nasal mucosa. The segment in the maxilla is usually cystic with the nasal and choanal segments are usually solid. ${ }^{1}$ Choanal polyps may also arise from the sphenoid sinus, the nasal septum, and other parts of the nasal cavity. ${ }^{2-4}$ The main symptom of presentation mainly of nasal obstruction. This can occur mostly unilaterally, although bilateral ACPs have been reported in literature. Antrochoanal polyps represent only $\sim 5 \%$ of sinonasal polyps. They are most commonly seen in young adults in their 3 rd to 5 th decades. They are found more commonly in males than females.

The exact etiology is not known. Infact this disorder is not associated with nasal allergy. Some of the postulated causes are association with an infective etiology. The association between antrochoanal polyp and chronic rhinosinusitis is also documented. ${ }^{6-8}$ Chronic sinusitis is present in $\sim 25 \%$ of patients but a causal relationship has not been firmly established. One among the other hypothesis are the Bernoulli phenomenon, whereby the negative pressure caused by the respiratory airflow perpendicular to the ostium, leading the polyp to extend into the nasal cavity. ${ }^{6,7}$ They occur more commonly among people who have allergies, cystic fibrosis, aspirin sensitivity, or certain infections. They are overgrowths of the mucous membranes. The growth of the polyp is due to impediment to the venous return from the polyp. This impediment occur at the level of the maxillary sinus ostium. This venous stasis increases the oedema of the polypoid mucosa thereby increasing its size.

Pathologically, antrochoanal polyps are identical to other inflammatory polyps. However, unlike other polyps, they usually have a narrow stalk arising from the maxillary sinus. ${ }^{5,7}$

Nasal endoscopy and computed tomography (CT) scans are required for making the diagnosis and for the planning of treatment. Radiographic diagnosis is usually done with the help of a non-contrast CT scan. However, in conventional X- ray od PNS, we can find a unilateral opacification of the maxillary sinus and nasopharyngeal mass. At times, we can observe a bilateral sinus involvement (23-42\%). CT scan is usually preferred as CT scan gives detailed information of the anatomy of paranasal sinus as well as extent of sinus involvement and anatomic variant.

Following are the features we can see in a non-contrast PNS CT 
examination which are best appreciated on true coronal or coronal reformatted scans:

i) A homogenous well defined mass arising from the maxillary antrum, with mucin density.

ii) The mass usually expand maxillary ostium and can be seen extending into the nasopharynx.

iii) Smooth enlargement of the maxillary sinus.

iv) No associated bony destruction of the surrounding bony structures.

However, antrochoanal polyps may demonstrate a higher density if they are long-standing and/or have an associated fungal infection. ${ }^{9}$ Contrast-enhanced scan may demonstrate peripheral enhancement. On an MRI, an intermediate to low signal on T1 and high homogeneous signal on T2 weighted images can be appreciated. Peripheral enhancement can be appreciated on post-contrast images. Impacted mucin/hemorrhagic content shows high signal in T1 weighted sequences.

\section{Differential diagnoses}

A. Acute sinusitis with edematous mucosa prolapsing from maxillary antrum.

B. Esthesioneuroblastoma which is a tumor arising from the superior recess of the nasal cavity.

C. Maxillary sinus mucocele which demonstrates complete opacification of the maxillary sinus associated with bony expansion.

D. Juvenile angiofibroma.

E. Nasal glioma.

F. Meningoencephalocele.

G. Inverted papilloma.

H. Mucocele.

I. Mucus retention cyst, Tornwalt's cyst.

J. Grossly enlarged adenoids.

K. Lymphoma and nasopharyngeal malignancies

\section{Management}

Treatment is done classically by intranasal snare polypectomy. The recurrence rates are usually low; however recurrence can be anticipated if the base of the stalk is not excised. Ideally with the help of minimally invasive endoscopic surgery, the attachment site of the stalk is identified and can be excised along with a small segment of the adjacent mucosa.

\section{Conclusion}

ACPs are benign polypoid lesions arising from the maxillary antrum and they extend into the choana. The commonest presenting symptoms are nasal obstruction and nasal drainage. ACPs should be considered in the differential diagnosis of unilateral nasal obstruction and a nasal mass. ACPs can be diagnosed by taking a careful history and conducting clinical exam as well as nasal endoscopic and radiological examinations. Physicians should focus on detecting the exact origin and extent of the polyp to prevent recurrence.

Antrochoanal polyps pose a challenge to the Otorhinolaryngologist. Many of its controversies pertaining to its pathogenesis and origin are solved and its nature and behavior known and the facts, established. Besides, various causative factors like infection in the anterior ethmoids, anatomically abnormal middle concha and septum, bacterial Infection of the sinus plays the key role, causing blockage to the acinous glands inside the antrum which is supposed to produce the cyst. This is one of the theories of formation of antrochoanal polyp. $\mathrm{CT}$ scan has got a better advantage compared to nasal endoscopy in detecting the anatomical variations, number of sinuses involved as well as to know the extent of disease in sinuses.

\section{Acknowledgements}

None.

\section{Conflict of interest}

Authors declare there is no conflict of interest in publishing the article.

\section{References}

1. Frosini P, Picarella G, de Campora E. Antrochoanal polyp: analysis of 200 cases. Acta Otorhinolaryngologica Italica. 2009;29(1):21-26.

2. Aydin O, Keskin G, Üştündağ E, et al. Choanal polyps: an evaluation of 53 cases. American Journal of Rhinology. 2007;21(2):164-168.

3. Kizil Y, Aydil U, Ceylan A, et al. Analysis of choanal polyps. Journal of Craniofacial Surgery. 2014;25(3):1082-1084.

4. Lessa MM, Voegels RL, Pádua F, et al. Sphenochoanal polyp: diagnose and treatment. Rhinology. 2002;40(4):215-216.

5. Towbin R, Dunbar JS, Bove K. Antrochoanal polyps. Am J Roentgenol. $1979 ; 132(1): 27-31$

6. Yaman H, Yilmaz S, Karali E, et al. Evaluation and management of antrochoanal polyps. Clin Exp Otorhinolaryngol. 2010;3:110-4.

7. Frosini P, Picarella G, De Campora E. Antrochoanal polyp: analysis of 200 cases. Acta Otorhinolaryngol Ital. 2009;29:21-6.

8. Ramesh C Deka. Antrochoanal Polyp: Its Pathogenesis Origin and Management by Functional Endonasal Endoscopic Surgery. Indian J Otolaryngol Head Neck Surg 1999;51(1):33-5.

9. Momeni AK, Roberts CC, Chew FS. Imaging of chronic and exotic sinonasal disease: review. AJR Am J Roentgenol. 2007;189(6):S35-45. 